Supporting Information

\title{
Interfacial Energy Barrier Tuning for Enhanced Thermoelectric Performance of PEDOT Nanowire/ SWNT/ PEDOT:PSS Ternary Composites
}

Siqi Liu, ${ }^{a}$ Junhua Kong, ${ }^{b}$ Haiming Chen, ${ }^{a}$ and Chaobin He ${ }^{*} a b$

a. Department of Materials Science and Engineering, National University of Singapore, 117574, Singapore. Email: msehc@nus.edu.sg

b. Institute of Materials Research and Engineering, A*STAR (Agency for Science, Technology and Research), 138634, Singapore. 
(a)

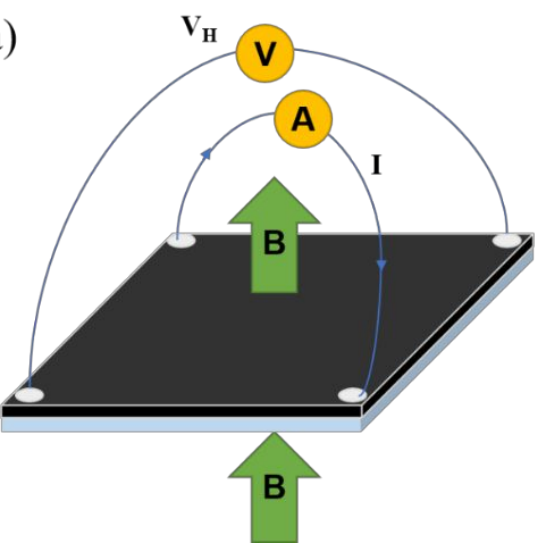

(b)

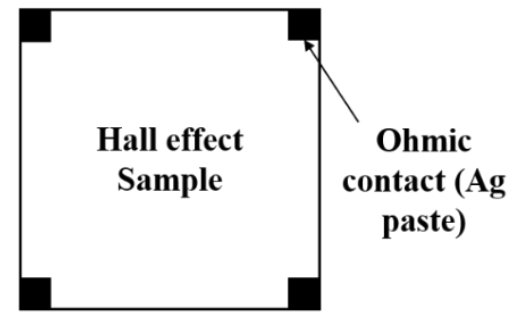

Figure S1. Schematic illustration of (a) the Hall effect and the measurement set-up (B is the magnetic field applied, $\boldsymbol{I}$ is the current applied and $\boldsymbol{V}_{\boldsymbol{H}}$ is Hall voltage) and (b) Van der Pauw geometry $(1.2 \mathrm{~cm} \times 1.2 \mathrm{~cm})$ used in this work. The measurement is conducted by HL5500PC Hall system, with $0.5 \mathrm{~T}$ magnetic field at room temperature.

By measuring the Hall voltage $\boldsymbol{V}_{\boldsymbol{H}}$, we can determine the sheet charge carrier density $\boldsymbol{n}_{\boldsymbol{s}}$ when knowing $\boldsymbol{I}, \boldsymbol{B}$ and elementary charge $\boldsymbol{q}\left(1.602 \times 10^{-19}\right)$ by the equation below, ${ }^{1}$

$$
n_{s}=\frac{I B}{q\left|V_{H}\right|}
$$

and the bulk charge carrier density is calculated by $\boldsymbol{n}=\boldsymbol{n}_{\mathfrak{s}} / \boldsymbol{t}$ if the sample thickness is known as $\boldsymbol{t}$.

Since the sheet resistance $\boldsymbol{R}_{\boldsymbol{s}}$ of the sample can be easily measured by Van der Pauw resistivity measurement, the charge carrier mobility can be extracted from the equation:

$$
\mu=\frac{\left|V_{H}\right|}{R_{S} I B}=\frac{1}{q n_{S} R_{S}}
$$




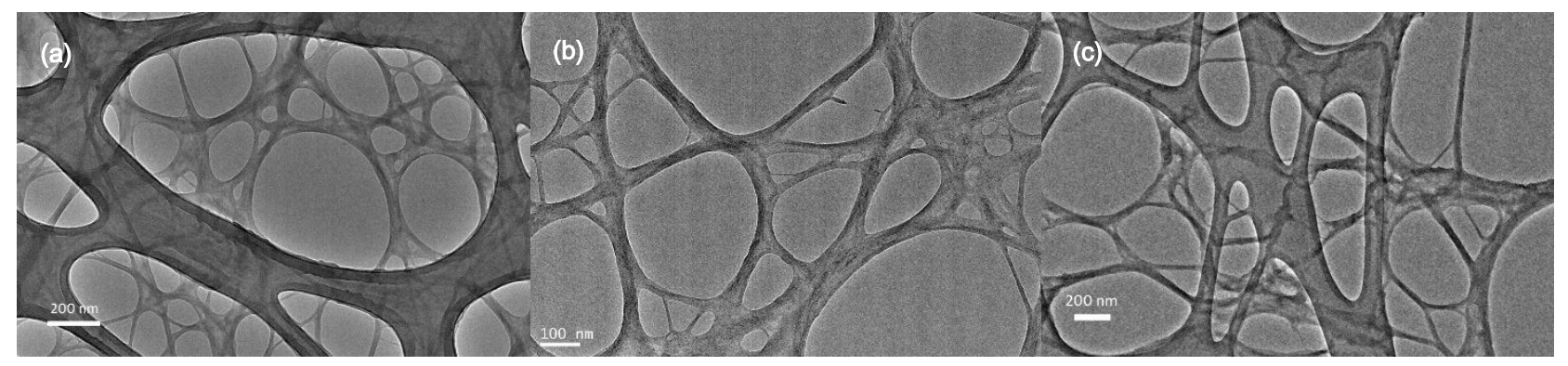

Figure S2. TEM images of as-synthesized PEDOT NW, (a) NW-0.214; (b) NW-0.858 and (c) NW-1.716 

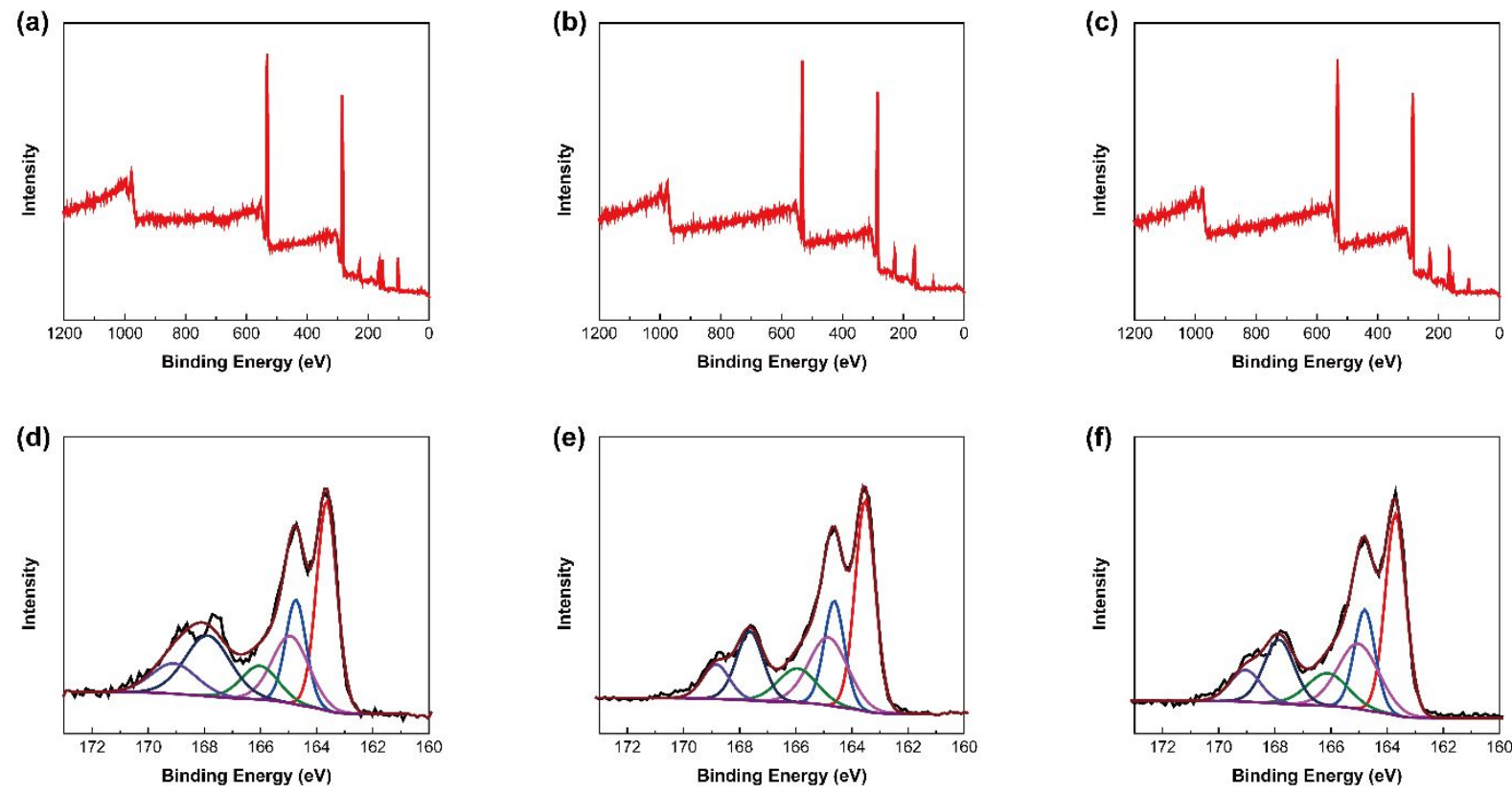

Figure S3. XPS survey and S2p core-level spectra of (a, d) NW-0.214; (b, e) NW-0.858 and (c, f) NW-1.716. 
Table S1. State of art of reported PEDOT:PSS-based TE composites.

\begin{tabular}{|c|c|c|c|c|c|c|}
\hline Materials & methods & loading (wt \%) & $\begin{array}{l}\mathrm{S} \\
\left(\mu \mathrm{V} \mathrm{K}^{-1}\right)\end{array}$ & $\begin{array}{l}\sigma \\
\left(\mathrm{S} \mathrm{cm}^{-1}\right)\end{array}$ & $\begin{array}{l}\mathrm{PF} \\
\left(\mu \mathrm{W} / \mathrm{mK}^{2}\right)\end{array}$ & Ref \\
\hline SWNT/PEDOT:PSS & Solution mixing & 85 & 20 & 3300 & 140 & 1 \\
\hline DWCNT/PEDOT:PSS & $\begin{array}{l}\text { Solution mixing then EG } \\
\text { treatment }\end{array}$ & 20 & 43.7 & 780 & 151 & 2 \\
\hline SWNT/PEDOT:PSS & $\begin{array}{l}\text { Solution mixing, DMSO } \\
\text { treatment }\end{array}$ & 6.7 & 59 & 1350 & 464 & 3 \\
\hline SWNT/PEDOT:PSS & $\begin{array}{l}\text { Solution mixing, } \mathrm{NaOH} \\
\text { treatment }\end{array}$ & 60 & 56.9 & 1701 & 526 & 4 \\
\hline Graphene/PEDOT:PSS & In-situ polymerization & 3 & 26.8 & 637 & 45.7 & 5 \\
\hline $\begin{array}{l}\text { MWNT/Graphene/PEDOT:PS } \\
\text { S }\end{array}$ & In-situ polymerization & $\begin{array}{l}3 \% \text { or } 5 \% \text { of } \\
\text { PSS solid } \\
\text { contents }\end{array}$ & 23.2 & 689 & 37.08 & 6 \\
\hline PEDOT:PSS/Bi ${ }_{2} \mathrm{Te}_{3}$ & Solution mixing & 4.1 & 1295 & 16 & 32.3 & 7 \\
\hline PEDOT:PSS/MoS 2 & Solution mixing & 4 & 1250 & 19.5 & 45.6 & 8 \\
\hline PEDOT:PSS/SnSe & Solution mixing & 20 & 320 & 110 & 380 & 9 \\
\hline PEDOT:PSS/Cu2Se NWs & Wet-chemical process & - & 1047 & 50.8 & 270.3 & 10 \\
\hline PEDOT:PSS/rGO/Te & $\begin{array}{l}\text { Wet-chemical and solution } \\
\text { mixing }\end{array}$ & - & 35 & 202 & 143 & 11 \\
\hline
\end{tabular}


Table S2. TE property of individual component SWNT, PEDOT:PSS-DMSO, PEDOT NW and binary composite SWNT-60 and ternary composite SWNT/PEDOT:PSS/PEDOT NW.

\begin{tabular}{cccc}
\hline & $\sigma\left(\mathrm{S} \mathrm{cm}^{-1}\right)$ & $\mathrm{S}\left(\mu \mathrm{V} \mathrm{K}^{-1}\right)$ & $\mathrm{PF}\left(\mu \mathrm{W} \mathrm{m}^{-1} \mathrm{~K}^{-2}\right)$ \\
\hline SWNT & 483.6 & 39.2 & 74 \\
PEDOT:PSS-DMSO & 719.3 & 23.9 & 41 \\
PEDOT NWs ${ }^{12}$ & 541.0 & 20.8 & 23 \\
SWNT/PEDOT:PSS (SWNT-60) & 2695.1 & 31.5 & 264 \\
SWNT/PEDOT:PSS/PEDOT NWs & 2503.5 & $\mathbf{3 7 . 5}$ & $\mathbf{3 5 2}$ \\
\hline
\end{tabular}




\section{Reference}

(1) Beer, A. C. The Hall Effect and Related Phenomena. Solid-State Electronics 1966, 9, 339-351.

(2) Moriarty, G. P.; De, S.; King, P. J.; Khan, U.; Via, M.; King, J. A.; Coleman, J. N.; Grunlan, J. C. Thermoelectric behavior of organic thin film nanocomposites. J. Polym. Sci. Part B: Polym Phys. 2013, 51, 119-23.

(3) Lee, W.; Kang, Y. H.; Lee, J. Y.; Jang, K. S.; Cho, S. Y. Improving the thermoelectric power factor of CNT/PEDOT:PSS nanocomposite films by ethylene glycol treatment. RSC Adv. 2016, 6, 53339-44.

(4) Hsu, J-H.; Choi, W.; Yang, G.; Yu, C. Origin of unusual thermoelectric transport behaviors in carbon nanotube filled polymer composites after solvent/acid treatments. Org. Electron. 2017, 45, 182-9.

(5) Liu, S.; Li, H; He, C. Simultaneous enhancement of electrical conductivity and seebeck coefficient in organic thermoelectric SWNT/PEDOT:PSS nanocomposites. Carbon. 2019, 149, 25-32.

(6) Yoo, D.; Kim, J.; Kim, J. H. Direct synthesis of highly conductive poly(3,4-ethylenedioxythiophene):poly(4styrenesulfonate) (PEDOT:PSS)/graphene composites and their applications in energy harvesting systems. Nano Res. 2014, 7, 717-30.

(7) Yoo, D.; Kim, J.; Lee, S. H.; Cho, W.; Choi, H. H.; Kim, F. S.; Kim, J. H. Effects of one- and two-dimensional carbon hybridization of PEDOT:PSS on the power factor of polymer thermoelectric energy conversion devices. J. Mater. Chem. A. 2015, 3, 6526-33.

(8) Du, Y.; Cai, K. F.; Chen, S.; Cizek, P.; Lin, T. Facile preparation and thermoelectric properties of $\mathrm{Bi}_{2} \mathrm{Te}_{3}$ based alloy nanosheet/PEDOT:PSS composite films. ACS Appl. Mater. Interfaces. 2014, 6, 5735-43.

(9) Jiang, F.; Xiong, J.; Zhou, W.; Liu, C.; Wang, L.; Zhao, F.; Liu, H.; Xu, J. Use of organic solvent-assisted exfoliated MoS2 for optimizing the thermoelectric performance of flexible PEDOT:PSS thin films. J. Mater. Chem. A. 2016, 4, 5265-73.

(10) Ju, H.; Kim, J. Chemically Exfoliated SnSe Nanosheets and Their SnSe/Poly(3,4ethylenedioxythiophene):Poly(styrenesulfonate) Composite Films for Polymer Based Thermoelectric Applications. ACS Nano. 2016, 10, 5730-9.

(11) Lu, Y.; Ding, Y.; Qiu, Y.; Cai, K.; Yao, Q.; Song, H.; Tong, L.; He, J.; Chen, L. Good Performance and Flexible PEDOT:PSS/Cu2Se Nanowire Thermoelectric Composite Films. ACS Appl. Mater. Interfaces. 2019, 11, 12819-29.

(12) Choi, J.; Lee, J. Y.; Lee, S. S.; Park, C. R.; Kim, H. High-Performance Thermoelectric Paper Based on Double Carrier-Filtering Processes at Nanowire Heterojunctions. Adv. Energy Mater. 2016, 6, 1502181.

(13) Zhang, J.; Zhang, K.; Xu, F.; Wang, S.; Qiu, Y. Thermoelectric transport in ultrathin poly(3,4ethylenedioxythiophene) nanowire assembly. Compos. Part B: Eng. 2018, 136, 234-40. 